UCRL-ID-123451

\title{
Passive Magnetic Bearings for Vehicular Electromechanical Batteries
}

\author{
Richard F. Post
}

March 1996

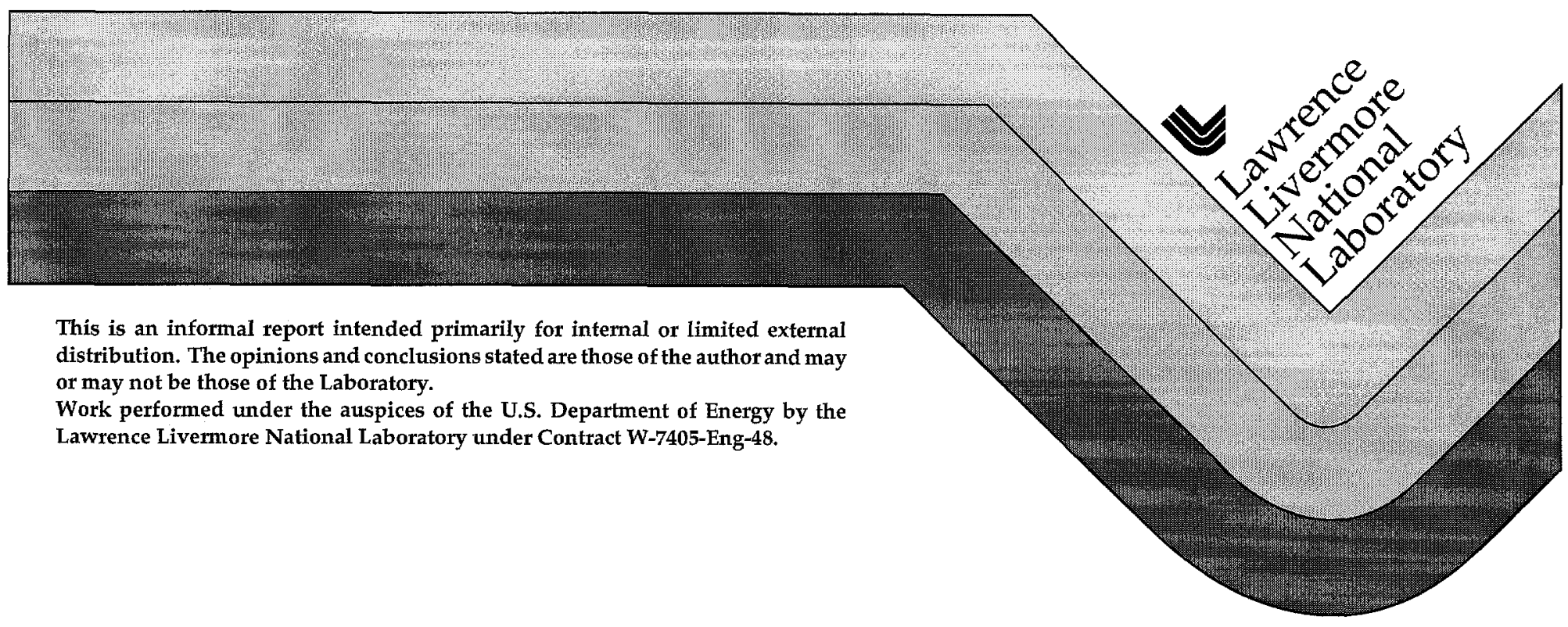



This document was prepared as an account of work sponsored by an agency of the United States Government. Neither the United States Government nor the University of California nor any of their employees, makes any warranty, express or implied, or assumes any legal liability or responsibility for the accuracy, completeness, or usefulness of any information, apparatus, product, or process disclosed, or represents that its use would not infringe privately owned rights. Reference herein to any specific commercial product, process, or service by trade name, trademark, manufacturer, or otherwise, does not necessarily constitute or imply its endorsement, recommendation, or favoring by the United States Government or the University of California. The views and opinions of authors expressed herein do not necessarily state or reflect those of the United States Government or the University of California, and shall not be used for advertising or product endorsement purposes.

This report has been reproduced directly from the best available copy.

Available to DOE and DOE contractors from the Office of Scientific and Technical Information

P.O. Box 62, Oak Ridge, TN 37831

Prices available from (423) 576-8401

Available to the public from the National Technical Information Service

U.S. Department of Commerce 5285 Port Royal Rd. Springfield, VA 22161 



\title{
Passive Magnetic Bearings for Vehicular Electromechanical Batteries
}

\author{
Richard F. Post \\ Lawrence Livermore National Laboratory
}

\section{I) Introduction}

This report describes the design of a passive magnetic bearing system to be used in electromechanical batteries (flywheel energy storage modules) suitable for vehicular use. One or two such EMB modules might, for example, be employed in a hybrid-electric automobile, providing efficient means for power peaking, i.e., for handling acceleration and regenerative braking power demands at high power levels. The bearing design described herein will be based on a "dual-mode" operating regime as follows:

The bearing/suspension system for use in a modular electromechanical battery that is intended for service in a hybridelectric drive automobile has two distinctly different service requirements. For the majority of its service life, say 100,000 hours (11.4 years) the vehicle will be parked and the bearing system must have low losses (typically $10 \mathrm{Watts}$ or less) and, preferably, operate in a contactless mode, that is, with pure magnetic levitation. By contrast, during the approximately 4000 hours of operation on the road $(100,000$ miles at an average speed of $50 \mathrm{mph})$ that the vehicle will experience during its service life the requirements are markedly different: Acceleration loads will be frequent and substantial, but increased bearing losses (say several tens of watts) during those momentary loads, as long as they are accommodated thermally, will be of little consequence as compared to the large power transfers occurring between the EMB and the drive train.

These considerations permit the design of a dual-mode bearing system, i.e., one that has low losses (and relatively low stiffness) when the vehicle is parked, but has high stiffness under acceleration overloads while the vehicle is on the road. The former objective can be achieved through the passive bearing concepts to be described in this report. The latter one can be accomplished by means of "backup" bearings whose expected service life thus needs only to be a few thousand hours. 
In the LLNL design of an EMB, because of its use of a dipole Halbach array in the generator/motor, the gap between the magnet array and the vacuum barrier between the array and the stator can be large. Also, as predicted by theory and as demonstrated in prior tests, there are virtually no sideways forces associated with the transfer of high peak powers out of or into the rotor. The consequence of these circumstances is that the passive bearing system can have relatively low stiffness and permit relatively large displacements before contact is made with the secondary (backup) bearing system. This relaxation of requirements makes it possible to design a viable passive magnetic bearing system for vehicular service with bearing stiffnesses that are much lower than those required in systems employing conventional generator/motor designs. In those systems the generator/motor system has narrow gaps and may exert large transverse, whirl-producing, forces, thus demanding both high bearing stiffnesses and strong damping for stable operation.

To accomplish the operational objectives that have been described the passive magnetic bearing system will use permanent magnet elements in its components. However, since the intensity of the magnetic field from such elements is temperature dependent, and since vehicular use involves a large range of ambient temperatures, this fact imposes an additional requirement on the design: The force equilibria and the behavior under accelerations of the bearing system should be relatively insensitive to changes in the remanent field of the permanent magnets caused by temperature variations. This requirement rules out a large class of bearing designs that would otherwise be satisfactory.

Summarizing, the passive magnetic suspension system to be described will have low losses when the vehicle is parked. When the vehicle is in motion the magnetic bearing system, possibly aided by shock-mounts, will have stiffnesses that are adequate to accommodate normal accelerations (for example, up to $1.0 \mathrm{~g}$ ). Beyond these levels it will employ a momentary-contact backup bearing system, as called for by acceleration loads that lead to large lateral or vertical displacements of the rotor relative to its housing. The losses of this secondary bearing system need not be lower than those set by thermal considerations, and its duty-cycle time will be a very small fraction of the service life of the vehicle. The overall design will also be shaped by the requirement that the performance of the bearing system should be adequately insensitive to changes in 
the parameters of its permanent magnets caused by changes in the ambient temperature.

\section{II) General Design Principles}

The general design principles involved in the LLNL passive magnetic bearing systems are described in the author's issued patent: "Dynamically Stable Magnetic Suspension/Bearing System.", U.S. Patent $\# 5,495,221$. The patent describes a variety of embodiments employing purely passive means for overcoming the strictures of Earnshaw's Theorem (the theorem that proves the impossibility of statically levitating objects by means of magnetic fields produced by fixed permanent magnets or fixed electrical currents). The concepts involved include:

- The use of dynamical effects to stabilize a rotating object above a low critical speed. (From rest and until this critical speed is reached mechanical or other means are used to maintain stability.)

- The achievement of stability (above a critical speed) by the tailoring of the vector sums of the force derivatives of the several elements of the bearing system so as to satisfy specific quantitative constraints.

- The use of spatially periodic magnetic fields, coupled with rotation, to produce a repelling force between two elements, and the use of such elements to provide stabilizing force derivatives.

The underlying thesis is that stable levitation of a rotor must be attained by a quantitative "tuning" of the force derivatives (negative or positive stiffnesses) of the several elements of a passive magnetic bearing system, where each element of the system, by itself, is unstable for either radial or axial displacements or, at best, is neutrally stable.

In the description to follow of a specific passive bearing design there are four types of elements that will be used to construct the entire system. They are:

1. Pairs of washer-shaped permanent magnet rings (or their equivalent) used in either an attracting or a repelling mode. 
2. Planar Halbach arrays [1], consisting of trapezoidal-shaped permanent-magnet bars arranged side-by-side to form a circular array.

3. Planar circuit arrays within which currents are induced, with the generation of repelling forces, when these arrays are near to a rotating Halbach magnet array.

4. Conventional ball-bearings, in mountings that engage at low rotation speeds, and then disengage at higher speed, leaving clearance gaps that define the maximum displacements before "touch down" occurs.

The bearing system as a whole will be built up from various combinations of the above elements, sized so as to be compatible with the requirements of levitation (force balance against gravity) and stability (net negative force derivatives).

\section{III) Geometry of the Bearing System}

The EMB with which the magnetic bearing system is to be used has a vertical axis of rotation. Thus the direction of the levitation force required is also vertical. However, when the vehicle is in motion, and unless some form of gimballing is employed, the rotation axis may tip a few degrees from the vertical, thus requiring both axial and radial components of levitating force. To provide the vertical levitating force in a manner that is intrinsically stable against axial displacements, i.e., where there always will exist an axial position that is at the minimum point of the axial potential function, we will employ two pairs of ring-shaped permanent magnets (or their equivalent), operating in a repelling mode. The uppermost element of the upper pair is fastened to the support structure, as is the lowermost element of the lower pair. Each of these stationary elements will be able to exert a maximum force on its rotating partner that exceeds the weight of the rotor. This requirement forces the position of axial force equilibrium to be located between the points on the axis corresponding to the maximum allowable displacements, up or down, before physical contact between the repelling magnet pairs occurs. This opposedpair configuration also satisfies another requirement that we have made, namely, insensitivity to ambient temperature changes. As long as the excess magnetic force from the exciting permanent 
magnets, alluded to above, persists at the maximum ambient temperature that is to be encountered, an axially stable equilibrium will always be maintained.

Thus far we have created a situation where stability against axial displacements is assured, independent of temperature or orientation of the axis. (It would work just as well upside down.) However, as dictated by Earnshaw's theorem, this system is unstable against displacements, either purely transverse to the axis of rotation, or tilting in nature. To stabilize the system against such displacements requires no net transverse force if the axis is vertical. It only requires the presence of elements that provide negative force derivatives in the transverse direction whose magnitude exceeds the positive (unstable) force derivatives associated with the top and bottom repelling element pairs. There is also no need for the added stabilizing elements to provide an axial force component. This force component is provided stably by the repelling pairs at top and bottom.

To provide the required radial stabilizing force derivatives, top and bottom, we will employ special passive bearing "cartridges." Each such cartridge is made up of three of the elements described above. At the top and the bottom of each cartridge there will be located ring-shaped magnet pairs (or their equivalent), oriented in the radially stable mode. The third element, needed for axial stabilization of the other two is described in detail below. It is composed of two Halbach arrays rotating above and below a stationary set of windings, as described in items 2 and 3 of the list of bearing elements given earlier.

Considering first the use of ring-shaped permanent magnets, there are two possibilities: One is to use a pair of matching-diameter annular magnet rings (or their equivalent) magnetized in the attracting mode, at the top and bottom of the cartridge. The inner members of each pair will be connected to the rotating shaft that supports the rotor, leaving a small gap at each end between the rotating magnets and their stationary partner. When the gaps are equal at both ends the axial attractive force exerted between the inner and outer magnets of the two magnet pairs will exactly cancel. At this (unstable) position of axial force equilibrium the cartridge will be capable of supplying a strong radial force derivative with no axial force component. The general configuration just described is shown schematically in Figure 1a. 
The second way in which the cartridge could be built using permanent magnet rings would be to use nesting annular ring magnets, with the direction of magnetization being the same in each. When this magnet pair is nested symmetrically with respect to each other it exhibits a strong radially restoring force derivative, with no axial force component. This configuration is depicted schematically in Figure $1 \mathrm{~b}$.

Because it will be used later in a "straw man" bearing design, another approach, one that uses soft iron poles in its operation, will be mentioned. Figure 2 illustrates a possible form this type of bearing element might take. As shown (in section) annular poles are excited by a washer-shaped ring of permanent magnet material embedded within the pole structure. The force (either attracting or repelling) that will be exerted between two similar pole structures arises from the magnetic fields in the radial gap between the inner and outer pole faces. This configuration, though more complicated than the use of simple ring-shaped magnets (as in Figures $1 \mathrm{a}$ and 1b), has two major advantages: First, the use of soft-iron poles will tend to smooth out any azimuthal variations in magnetic field strength associated with azimuthal non-uniformity of the properties of the permanent magnet ring. This smoothing effect will lead to reduced eddy current losses. The second advantage is that the pole structure provides a means for strengthening the assembly against centrifugal forces. High-field permanent-magnet material, though strong in compression, is rather weak in tension. In the magnet-ring design the forces in the rings is tensile in nature. However, in the pole design the forces on the ring magnet (which may be segmented azimuthally) are compressive, allowing higher rotation velocities for a given bearing element diameter. Furthermore, if need be the outer surface of the outside pole can be reinforced by winding it with highmodulus graphite fiber composite.

Using only any pair of the type of elements described above, however, the axial force equilibrium position within the cartridges is an unstable one. That is, in each case the axial position of equilibrium is characterized by negative stiffness (positive force derivative). Again, however, no net axial force is required to maintain this equilibrium; only a net negative (restoring) force derivative is required. The negative force derivative required for internal axial stability of each cartridge is to be supplied by a special arrangement of two planar Halbach arrays, attached to the shaft, 
coupling magnetically to a stationary planar circuit array located midway between them. This configuration is shown schematically in Figure 3.

A further essential feature of the idea is that the Halbach arrays must be oriented relatively to each other azimuthally "in phase," that is, so that their azimuthal field components add at the midplane, while their axial field components cancel. When this is the case, and when the planar circuit array is located midway between the two Halbach arrays, there will be no induced voltages and currents in the planar circuits, thus no resistive power losses. (There will be small eddy-current losses in the conductors. These losses can be minimized by the use of litzendraht wire for the conductors.) However, as soon as an axial displacement, either up or down, occurs, strong restoring forces will appear that will re-center the rotating magnet pairs. Theory shows that substantial axial force derivatives can be obtained from this configuration, even with quite small Halbach arrays. All that is required is that this (negative) force derivative should be larger than the combined (positive) axial force derivative associated with the two magnet pairs in the cartridge.

Again, it should be recognized that the cartridge concept described here is one that is insensitive to temperature changes (as long as they are about the same for all the permanent magnet elements in the cartridge). This insensitivity occurs because of the balanced-force nature of the axial equilibrium position in the cartridge, for either of the two embodiments.

The cartridges described also have two additional elements that perform necessary functions. When the rotor is at rest, or when its speed is below a critical speed, the Halbach array system will not be able to exert its axially stabilizing influence on the axially unstable. elements in the cartridge. In the embodiment using two magnet rings (or two iron poles) facing each other, the inner two magnets (or poles) of the two pairs will be unstable axially and will move so as to close one or the other of the gaps between them and their stationary mate. In the second embodiment, the one employing concentric permanent magnets (or their equivalent with nesting pole structures) the equilibrium position is unstable in that the inner magnet will try to pop out axially from its centered position. We will take advantage of this effect, in either embodiment, to engage ball bearings, one at each end of a cartridge. Axial motion beyond the excursions normally expected in operation will result in socketing the 
outer ball bearing race in a recessed hole, thus limiting both axial and radial displacements. Now, when the rotor is speeded up above its transition speed (substantially below its normal operating speeds), the Halbach arrays will generate an axial force sufficient to center the system, thus disengaging the ball bearing, whichever one is engaged. In the disengaged position, however, the ball bearings will still be surrounded by a shroud ring with a clearance that defines the displacement at touchdown. The ball bearings thus provide two functions: (1) axial stabilization of the cartridges below the transition speed, and (2) touchdown bearings as required for radially or axially directed accelerations beyond the level of those that can be accommodated by the axial or radial magnetic bearing elements.

With care in the design, and in the symmetrization of the levitating and centering permanent magnet elements (the washerlike elements or their equivalent), there should only be low power losses (those arising from eddy currents in the litzendraht wire) from these elements. Also, as we have mentioned, when the cartridges are operated in a centered position, there will be minimal resistive losses in the planar circuit arrays. To minimize losses when the vehicle is parked it remains to insure that this centered condition is maintained. The corollary is that neither cartridge should be called upon to provide an appreciable axial force component when the vehicle is parked, or during normal driving. During normal driving (or in the infrequent case when large vertical accelerations are encountered) it will be required that the associated vertical forces on the rotor are either supported by the repelling bearing elements, or by the backup ball bearings. The way that this objective can be accomplished is through the design of the support structure for the bearing cartridges. The cartridges are to be supported by springs and/or guides that constrain their motion radially, while allowing them to move axially about their equilibrium position freely. A simple example of such a mount would be two or more vertical rods sliding in holes in the cartridge housing, with low compliance springs supporting the cartridges against gravity. Within defined vertical limit stops the cartridges would bob up and down freely under normal acceleration loads, with the axial forces on the rotor (arising from vertical accelerations) being taken up by the repelling bearings. On the occasion of encountering a pot hole the displacement would be large enough to make the cartridges hit their vertical stops, thus bringing the backup bearings into play. 
A schematic drawing illustrating the main features of a bearing cartridge and its component parts is shown in Figure 4. In the example shown the attracting magnet pairs are annular permanent magnets in the configuration described earlier (Figure 1a).

Because of the use of guide rods and low compliance springs to support the cartridges, the force exerted on the Halbach arrays by the planar circuits will always be limited to that required to keep the cartridge centered relative to its rotating elements as the rotor moves up and down. Since the mass of the cartridges will be very small compared to that of the rotor, the axial forces required to maintain centering under normal conditions (i.e., for displacements less than those defined by the vertical stops) will be correspondingly small. At no time will the planar circuit be called on to deliver a large force. When large forces are required (the pot-hole), the backup bearings will take the load. Limiting the vertical force required of the planar circuits will correspondingly limit the power dissipation in those circuits to a low value under all circumstances.

\section{IV) Quantitative Considerations: Rotor Parameters}

To perform a detailed design of a bearing system based on the concepts outlined above would require analysis of a specific system. In the absence of such data, however, there are some general analytical results and scaling laws that can be set down. These results will aid in assessing the design issues to be addressed in carrying out a detailed design. To normalize these results we will use as a "straw man" case an EMB with the following specifications:

- Usable stored energy (with 2:1 speed ratio)

$0.5 \mathrm{kWh}$

- Peak power of generator/motor

$50 \mathrm{~kW}$

- Aver. energy density in fiber comp. (at full speed) $200 \mathrm{Wh} / \mathrm{kg}$

- Weight of rotor (fiber composite only) $3.33 \mathrm{~kg}$

- Weight of Halbach array + rotating bearing elements $1.67 \mathrm{~kg}$

Of these specifications the critical one is the total levitated weight, $5.0 \mathrm{~kg}$., from which the major parameters of the magnetic bearing system can be defined. The only important parameter left undefined by the above table of values is the maximum operating 
speed of the rotor. The peak rotation speed will be determined by the aspect ratio of the rotor itself, i.e., whether it is it is ring-like, or in the form of an elongated cylinder. If ring-like, the peak rotation speed, being determined by the peripheral speed of the rotor, would be typically in the range of 30 to 40 thousand RPM. If cylinder-like, the speed would be higher, say 75 to 90 thousand RPM. The bearing design sketched in what follows should, however, not be particularly sensitive to which one of these two speed ranges is applicable.

Returning to the issue of determining the required levitating forces, it is necessary to define the peak " $\mathrm{g}$ " loads that are to be borne by the magnetic elements (before the mechanical backup bearings come into play) Assuming that shock-absorbing mounts are used (in addition to the vehicle's shock absorbers) to filter out high-frequency components of road-induced accelerations, we will here specify that the maximum acceleration loads to be sustained by the magnetic elements are limited to $1.0 \mathrm{~g}$, i.e., to acceleration-induced forces of order 50 Newtons. The direction of these accelerations might be either primarily vertical (as in going over a bump in the road), or primarily horizontal, as in "panic-stop" braking. Acceleration loads beyond this level, as noted, would be taken up by the backup bearing system.

In addition to the levitating force requirements, the magnetic bearing system will be subject to other requirements. One of these is that its design should be such as to minimize losses of eddy-current origin, subject to the accompanying economic constraint that the permanent magnet material used in its manufacture should not be required to adhere to unduly rigid standards of uniformity of magnetization. The origin of the need to reduce eddy current losses comes from two sources: (1) the need to minimize problems of thermal management, and, (2) the requirement for an adequately long rundown time for the EMB when the vehicle is parked. For the purposes of the straw man design given here, we will consider that total eddy current losses in the bearing system of 5 Watts or less would be acceptable. That loss level would correspond roughly to a run-down time constant of about 100 hours for the $0.5 \mathrm{kWh}$ system we are considering (assuming that losses of aerodynamic origin are made to be negligible by good vacuum practice in the design of the rotor housing). In this report it will not be possible to exactly specify eddy-current losses, in the absence of experimental data. However, it is believed on the basis of the approximate analysis given that the concepts described permit the design of a bearing system that should have losses that are at least as low as 5 Watts. It is possible that they might be made to be 
much lower than this figure if sufficient care is taken in the actual implementation of the design.

\section{V) Quantitative Considerations: Bearing Scaling Laws}

The design of a bearing system meeting specifications such as those given above can be facilitated by taking advantage of simple scaling laws. The use of these laws allows one to scale forces, force derivatives, and bearing weights as a function of the bearing element radius alone, using data from any attracting or repelling element for which these parameters are known. As long as all the appropriate dimensionless parameters, such as the ratio of magnet thickness to magnet radius, the ratio of magnet separation (between two magnets) to magnet radius, etc., are kept constant, then in the scaled dimensions the strength of the magnetic field will be the same as before. Since the force between the magnet elements varies as the product of an area and the square of the strength of the magnetic field, it follows that the force will be proportional to the radius of the magnet element, squared. That is, at the same dimensionless separation (i.e., the separation measured in units of the radius), for the original magnet pair (subscript 1 ) and the scaled pair (subscript 2) the ratio of their forces will scale as:

$$
\frac{F_{2}}{F_{1}}=\left[\frac{a_{2}}{a_{1}}\right]^{2},
$$

where $a_{2}$ and $a_{1}$ are the respective radii of the two magnet pairs.

It follows that at the same dimensionless separation between the magnet pairs, the derivative of the force (in the axial direction) is linearly proportional to the ratio of the radii, i.e.,

$$
\frac{\mathrm{dF}_{2} / \mathrm{dz}}{\mathrm{dF}_{1} / \mathrm{dz}}=\left[\frac{\mathrm{a}_{2}}{\mathrm{a}_{1}}\right]
$$

Since for axially symmetric elements the force derivative (for small displacements in the radial direction) is just one-half of the force derivative in the axial direction, it follows that the force derivative in the radial direction scales in the same way with radius of the magnet elements as that for the axial force derivative. 
The final relationship that we need, for economic reasons, for example, is the scaling law relating the weights of the bearing elements. This relationship is, of course, the simple one:

$$
\frac{M_{2}}{M_{1}}=\left[\frac{a_{2}}{a_{1}}\right]^{3}
$$

Since the three parameters we have listed scale in different ways with size of the bearing element their relative values will of course change with scaling. In the case of the force and its derivatives we see that the force derivative increases, relative to both the force itself, and relative to the weight of the bearing element, as the radius of the element is decreased. If increased stiffness (negative force derivative) is advantageous, this circumstance may influence the design of the overall bearing system. That is to say, the replacement of a single bearing element pair by two same-shaped smaller ones exerting the same total levitating force would result in a bearing system that has 1.414 times the stiffness in its stable direction (radial or axial) with a total weight 0.707 times that of the single pair. Of course it should also be recognized that for a given bearing element pair the ratio of the force derivative to the levitating force is a function of the separation between the pair. This circumstance provides the designer with another option for the control of this parameter, but obviously requires data concerning the particular elements involved and therefor cannot be reduced to a simple general scaling law.

An illustration of this latter method of scaling is the following: In the case of the bearing "cartridge" concept that we have outlined above there is a simple way in which to achieve any desired radial stiffness (within the bounds of the maximum value of this parameter for the elements themselves): This approach is to adjust the gaps of the two opposing elements to smaller or larger values. In this manner the radial force derivative is increased or decreased, respectively, without influencing the axial force balance of the bearing system as a whole. Increasing the radial stiffness reduces the radial displacement for a given lateral acceleration load, but at the same time puts additional demands on the axial stabilizer element that must overcome the increased axial negative stiffness that comes with increased radial positive stiffness. 
Generally speaking, in the absence of other restraints, wherever possible one should aim for the lowest radial stiffness that is tolerable, i.e., to aim at a design having a stiffness that is just adequate to restrain the rotor laterally under the maximum acceleration load to be sustained before the mechanical backup bearing system comes into play. In this way the least demands are put on the axial stabilizer, simplifying its design and leading in most cases to reduced residual power losses in this element.

Another reason for designing in the direction of smaller radial stiffnesses has to do with the eddy-current losses in the bearing elements themselves: If the magnetic fields of the bearing elements are sufficiently close to axial symmetry to minimize eddy-current losses from this origin when these elements are rotating concentrically, there is still another potential source of such losses. This source is the one associated with unbalance-related eccentricity in the axis about which the system rotates at high speeds (relative to the geometric axis of the bearing elements). High radial stiffness implies steep radial magnetic gradients in the magnetic gap between the elements. Steep gradients in this region imply increased eccentricity-induced periodically varying magnetic fields at the surface of the magnets themselves (if simple pairs of ring magnets are used), or at the pole faces of iron pole structures if these are used. These time-varying magnetic fields then will result in eddy-current losses in these metallic elements.

On the basis of simple estimates given below it is believed that with care in the design and with attention to the issue of balancing eddy-current losses will be quite small. However, if these losses present an appreciable problem the use of pole structures fabricated from powdered iron core material such as that used in the electronics industry would reduce eddy current losses to negligible levels.

\section{VI) Bearing Specifications for the "Straw man" Design}

Before discussing the dimensional and other parameters of a bearing design suitable for the "straw man" EMB we need better to define its requirements. The levitation requirement, $5 \mathrm{~kg}$, has already been specified and together with it, the maximum axial and radial excess force requirements, 50 Newtons, needed to satisfy $1.0 \mathrm{~g}$ acceleration loads, either horizontal or vertical. Still needed is a specification of the maximum allowable transverse displacement of the rotating elements of the bearing cartridges. That is, that displacement 
that would result in contact with the backup bearings (needed for infrequent higher-g acceleration loads). As noted earlier, typically there will exist a relatively large magnet-to-stator-housing gap in an EMB employing a dipole Halbach array. In light of this fact it seems reasonable to design for a maximum lateral displacement of $2 \mathrm{~mm}$. Combining this figure with the 50 Newton transverse force requirement leads to a required minimum total net positive radial stiffness of $2.5 \times 10^{4}$ Newtons/meter (140 $\mathrm{lbf} / \mathrm{in}$.). That is, the vector sum of the positive radial stiffness of the two bearing cartridges and the negative radial stiffnesses of the two repelling bearing elements should exceed this value.

In the vertical direction even larger displacements should be allowable, so that substantially lower stiffnesses would still be acceptable. In this case the axial stiffnesses are solely those associated with the two repelling elements, since the bearing cartridges, by the way they are mounted and constructed, do not contribute to the axial stiffness. Thus to satisfy the requirement on vertical stiffness we will therefor make the conservative assumption that a total vertical stiffness (the sum of the bottom and top repelling bearing stiffnesses) of the same magnitude as the total radial stiffness will be adequate.

\section{VII) A Bearing Design Using Annular Pole Assemblies}

Since force data from computer-code calculations of a particular annular pole assembly of the type shown in Figure 2 exists, we will use these data, together with the scaling laws given in Section $V$, to design the main elements of a complete bearing system for our straw man hybrid EMB system. Figure 5 depicts this same structure, but with call-outs for the various dimensions involved. The magnet material shown on the drawing is $\mathrm{NdFeB}$ with a remanent field of 1.25 Tesla. Using the dimensions and materials shown computer-code calculations give the the force as a function of axial displacement as shown in Figure 6 (attracting mode) and Figure 7 (repelling mode). Whether the pole faces attract or repel each other depends, of course, on the relative direction of magnetization (in the axial direction, as shown) of the permanent-magnet material.

Note first that the forces involved for bearing elements of the size indicated in Figure 5 are far larger than those needed for the small rotor weight of our straw man design. For example, the maximum repelling force, shown in Figure 7 , is over 400 Newtons, a force large enough to levitate over 40 kilograms of weight. 
In like manner, from Figure 6 we can estimate that for the attracting mode the radial force derivative from a single pair of elements is of order $(1 / 2) \times\left(1.5 \times 10^{6}\right)=7.5 \times 10^{5}$ Newtons/meter at a pole-to-pole gap (axial separation) of order $0.5 \mathrm{~mm}$. This magnitude of force derivative is 30 times larger than the value needed to satisfy the minimum requirement stated in Section VI, giving us considerable latitude in carrying out the overall design, where the vector sum of the radial force derivatives meeting those requirements is to be satisfied. As will be seen the desired value of radial force derivative can be achieved by a combination of scale-down and operation at a larger dimensionless axial gap. For example, at a gap of $2.5 \mathrm{~mm}$. the radial force derivative of the attracting pair has fallen by a factor of about 5 , to $1.5 \times 10^{5} \mathrm{~N} / \mathrm{mm}$. and at larger relative separations the force derivative will be even smaller.

Since the repelling pair will define the magnitude of the negative radial stiffness that must be overcome by the radially stabilizing bearing cartridges we must start our design with the former elements. We begin by choosing a median gap of $2.0 \mathrm{~mm}$ in Figure 7 for which the repelling force is about 300 Newtons and the axial force derivative is about $5.0 \times 10^{4}$ Newtons/meter. Over the range between $0.5 \mathrm{~mm}$. and $3 \mathrm{~mm}$. the force curve is seen to be approximately linear. At 0.5 $\mathrm{mm}$. the axial force is over 400 Newtons. We will take advantage of this increase in force at small displacements in the design, as a means of limiting the vertical displacement under vertical acceleration loads.

Since the full-scale bearing elements can levitate a much larger weight than our 5 kilograms, the next step is to choose the scale-down factor for the repelling bearing pair. Since the net vertical force that supports the rotor against gravity is the resultant of two opposing forces, up from the lower repelling pair and down from the upper pair, the position of the vertical equilibrium must reflect this fact. Here we have two choices: Either the upper and lower pairs could be identical, so that the equilibrium position is at a smaller vertical displacement for the lower element than the upper one, taking advantage of the variation of repelling force with displacement. Alternatively, the lower element could be larger in dimension than the upper, so that at the same dimensionless displacement the net upward force would be in equilibrium with the levitated weight. For the design sketched here we will choose the former of the two alternatives. 
From Figure 7 again, we will choose for the displacement at equilibrium of the lower repelling pair the value of $0.1 \mathrm{~cm}$, and for the upper pair a displacement of $0.3 \mathrm{~cm}$., corresponding to a difference force of about 150 Newtons. This force is about 3 times the levitating force required for our straw man EMB rotor. We will therefore need to scale down the size of the bearing elements (from that shown in Figure 5) by a factor $\sqrt{1 / 3}=0.577$ to arrive at our required levitating force. As a result of this scaling the axial stiffness is scaled down by the same factor. Approximating the slope of the repelling force curve as a linear function over the displacement range of $1.0 \mathrm{~mm}$. to 3.0 , the axial force derivative for one element pair is, before scaling down, equal to $-(400$ $-260) / .002=-7.0 \times 10^{4}$ Newtons/meter. The scaled down value is therefor equal to $-4.0 \times 10^{4} \mathrm{~N} / \mathrm{m}$. The axial stiffness of the upper and lower repelling elements combined is twice that value, i.e., $8.0 \times 10^{4}$ $\mathrm{N} / \mathrm{m}$.

With the above-indicated axial stiffness, a vertical accelerationinduced load of 50 Newtons (1.0 g vertical acceleration) would then result in a relative displacement (from the equilibrium position) of $50 /\left(8.0 \times 10^{4}\right)=6.25 \times 10^{-4} \mathrm{~m} .=0.6 \mathrm{~mm}$. Displacements resulting from upward accelerations larger than this would be restrained by the backup bearings.

Since the radial stiffness for small displacements of each repelling pair is negative one-half of that for axial displacements, the total negative radial stiffness that must be overcome by the two bearing cartridges is equal to $4.0 \times 10^{4} \mathrm{~N} / \mathrm{m}$. This figure then will determine the scaling factor that we use in designing the bearing cartridges, considered next.

For our design we will take as the radial force derivative of a magnet pair (before scaling down) as that associated with a $2.5 \mathrm{~mm}$. gap between the two poles, namely, $-1.5 \times 10^{5} \mathrm{~N} / \mathrm{m}$. Since there are two bearing cartridges, each having 2 pole pairs, the total radial force derivative (before scaling) is $-6.0 \times 10^{5} \mathrm{~N} / \mathrm{m}$.

We next calculate the actual radial force derivative that is required to be supplied to overcome the negative stiffness of the repelling bearings, plus the net positive stiffness requirement for the assembly as a whole, namely, $2.5 \times 10^{4} \mathrm{~N} / \mathrm{m}$. The negative radial stiffness introduced by the repelling elements, given above, is $4 \times 10^{4}$ 
$\mathrm{N} / \mathrm{m}$. It then follows that the cartridges must supply $\left(4 \times 10^{4}+2.5 \times\right.$ $\left.10^{4}\right)=6.5 \times 10^{4} \mathrm{~N} / \mathrm{m}$. to satisfy the radial stiffness requirement. From this fact we deduce that the scale factor for the radial force derivative of the attracting elements is equal to $\left(6.5 \times 10^{4} / 6.0 \times 10^{5}\right)=0.11$ From Equation 2 we see that this is the same factor by which the radius of the bearing must be scaled, so that we find for this radius the approximate value $a_{2}=6.0 \times .11=0.7 \mathrm{~cm}$., a small sized unit indeed. It is in fact so small that it may be worthwhile, to facilitate construction and assembly, to design to a larger displacement gap, off the end of the curve in Figure 6, to reduce the radial stiffness (before scaling) and thus permit the use of a bearing element with a larger radius.

To recapitulate, the radius of the repelling bearing elements is $6.0 \times .577=3.5 \mathrm{~cm}$., and the radius of the attracting bearing elements in the cartridges is $0.7 \mathrm{~cm}$. This combination of elements provides levitation and also provides both axial and radial stiffnesses sufficient to limit the radial and axial excursions of the rotor under acceleration loads of $1.0 \mathrm{~g}$ to $2.0 \mathrm{~mm}$. or less. In the next section we will consider the last major element in the overall design, namely, the design of stabilizer needed to overcome the negative axial stiffness associated with the two attracting elements in each of the two bearing cartridges.

\section{VIII) Design of the Axial Stabilizer}

As noted earlier, the axial stabilizer bearing element utilizes a planar array of litzendraht wire conductors sandwiched between two planar Halbach arrays to produce a strong positive axial stiffness when rotating, with near-zero power losses in the equilibrium position (planar conductor array midway between the two Halbach arrays). We will present here some approximate design equations for the axial force derivative derivable from such a system. These will serve to provide a first cut at a design for the stabilizer. A final design would require model tests to set the correct parameters. However, as will be seen the approximate equations may be adequate to set the dimensional scale of the arrays, with the separation between the two Halbach arrays being an adjustable parameter than can be used to "tune" to the desired performance.

We present below an equation giving an analytical result, useful for an approximate estimate of the force derivative achievable from a stabilizer, assuming it is operating well above its "transition speed". 
The transition speed is the speed where the inductive reactance of the circuit array, rising with rotation frequency, becomes equal in magnitude to its resistance. This transition typically occurs at a rotation speed that is much lower than the operating speed range of the EMB rotor. For example, for the case analyzed below it is estimated be about 5000 RPM, as compared to typical operating speeds of 40,000 to 80,000 RPM, depending on rotor design and aspect ratio.

The result for the axial force derivative is:

$$
\frac{\mathrm{dF}_{\mathrm{z}}}{\mathrm{dz}}=-\mathrm{N}_{\mathrm{c}}\left[\frac{2 \mathrm{r}_{2}^{2} \mathrm{~B}_{0}^{2}}{\mathrm{~L}_{0}}\right]\left[1-\frac{\mathrm{r}_{1}}{\mathrm{r}_{2}}\right]^{2}\left[1+\frac{\mathrm{r}_{1}}{\mathrm{r}_{2}}\right] \exp (-2 \mathrm{kh}) \quad \mathrm{N} / \mathrm{m}
$$

Here $r_{2}\left(m\right.$.) is the outer radius of the planar Halbach array and $r_{1}(m$.) is its the inner radius, $B_{0}$ (Tesla) is the strength of the magnetic field at the surface of the Halbach array (defined below, Equation 6), $N_{c}$ is the number of radially directed conductors in the planar circuit array and $\mathrm{L}_{0}$ (hy) is the inductance of a single loop of that circuit array (including both self- and mutual-inductance). The quantity $2 \mathrm{~h}(\mathrm{~m}$.) is the separation between the upper and lower Halbach arrays, and the parameter $\mathrm{k}\left(\mathrm{m}^{-1}\right)$ is the azimuthal wavenumber of the Halbach array, defined by the equation:

$$
\mathrm{k}=\mathrm{m} / \mathrm{r}_{2} \quad \mathrm{~m}^{-1}
$$

where $\mathrm{m}$ is the number of periods (wavelengths) around the Halbach array.

From Halbach's theoretical work [1] we have an expression for $\mathrm{B}_{0}$ (Tesla), the strength of the magnetic field at the surface of the Halbach array, given in terms of $B_{r}$, the remanent field of the permanent magnet material, the number of magnet sectors per azimuthal wavelength, $M$, and $d(m$.$) , the axial thickness of the sector$ magnets of the Halbach array:

$$
B_{0}=B_{r}[1-\exp (-k d)]\left[\frac{\sin (\pi / M)}{\pi / M}\right] \text { Tesla }
$$


(A typical value calculated for $B_{0}$ when using $\mathrm{NdFeB}$ magnet material with $B_{r}=1.25$ Tesla and taking $M=4$ and $k d ~>1$ is about 1.0 Tesla).

To illustrate the fact that, using Halbach arrays with a relatively small outer radius, it is still possible to generate stabilizing axial force derivatives at a substantial level, we will introduce the following values into equation (4):

We take $\mathrm{N}_{\mathrm{c}}=48, \mathrm{r}_{2}=.025 \mathrm{~m}$. and $\mathrm{r}_{1} / \mathrm{r}_{2}=1 / 3, \mathrm{~B}_{0}=1.0$ Tesla, $\mathrm{L}_{0}=$ $0.2 \times 10^{-6} \mathrm{Hy}$ (a typical value for planar circuits of this size with ferrite bead inductive loading), and $\mathrm{m}=2$ azimuthal wavelengths, yielding $\mathrm{k}=$ $2 /(.025)=80 \mathrm{~m}^{-1}$. Using these values we find for the axial force derivative the estimate:

$$
\left.\frac{\mathrm{dF}_{\mathrm{z}}}{\mathrm{dz}}=1.8 \times 10^{5} \exp (-160 \mathrm{~h})\right] \quad \mathrm{N} / \mathrm{m} .
$$

Recall now that the each axial stabilizer needs to contribute a positive axial stiffness that overcomes the negative axial stiffness of the attracting elements in a bearing cartridge. From the previous calculations we recall that the radial stiffness of each cartridge needs to be equal to $\left(6.5 \times 10^{4}\right) / 2=3.25 \times 10^{4} \mathrm{~N} / \mathrm{m}$ to overcome the negative axial stiffness of the repelling elements, plus the radial stiffness required to limit the transverse displacement of the rotor to $2 \mathrm{~mm}$. under acceleration loads of $1.0 \mathrm{~g}$. However, since the axial force derivative of the attracting elements is, in magnitude, just twice that of the radial force derivative the end result is that each axial stabilizer needs to contribute slightly in excess of $6.5 \times 10^{4} \mathrm{~N} / \mathrm{m}$. From Equation 7 above we see that this result can be achieved by making the separation, $2 \mathrm{~h}(\mathrm{~m}$.$) , between the two Halbach arrays equal to or$ slightly less than $1.2 \mathrm{~cm}$.

While the above results are only approximate they serve to illustrate that relatively small axial stabilizers $(5 \mathrm{~cm}$. in diameter in the example) can provide an adequate amount of positive axial stiffness to stabilize the axial instability of the pair of attracting elements in the cartridges. This even includes the additional stiffness implied by the fact that the radial stabilizers must provide sufficient stiffness to resist $1.0 \mathrm{~g}$ transverse loads while at the same time overcoming the negative radial stiffness of the repelling elements. 
The intent of the above exercise has not been to come up with a final design for a passive bearing system for an engineered EMB for hybrid vehicle service. It has instead been intended to provide an example that shows the overall feasibility of the concepts described. This feasibility relates both to meeting quantitative requirements such as acceleration loads, and to the fact that the system is made up of simple elements of small size, ones that should be able to be engineered and fabricated at modest cost.

In connection with the cost issue, it would appear that the only elements of the system whose cost per kilogram will be a consideration is that of the $\mathrm{NdFeB}$ magnets, projected to be about $\$ 50 / \mathrm{kg}$. From the data we have given above on sizes of the elements and using the scaling law, Equation 3, for the scaling laws, we can estimate the total weight of $\mathrm{NdFeB}$ that would be required in the "straw man" design that was presented. The results are as follows.

Total weight of (8) magnets in the attracting elements: $.003 \mathrm{~kg}$

Total weight of (4) magnets in the repelling elements: $.25 \mathrm{~kg}$

Total weight of (4) magnet sets in the Halbach arrays: $\quad .54 \mathrm{~kg}$ $\overline{.80 \mathrm{~kg}}$

Examining this table it can be seen that it probably would be worthwhile to increase the size of the attracting elements, while working at a larger dimensionless gap, so as to make their construction less of a "watchmakers job", since their contribution to the magnet cost at the indicated size is so small. Other optimizations could include:

(1) Reducing the thickness of the Halbach array magnets, thus their weight, by operating with a narrower gap between them. For example, reducing the thickness of the magnets by a factor of 2 , while closing the gap between the magnet arrays to $0.6 \mathrm{~cm}$. would result in the same axial stiffness at one-half the weight of magnets.

(2) Reducing the weight of the magnets in the repelling elements by allowing a larger vertical displacement before contacting the backup bearings (displacements in the axial direction can be much larger than $2 \mathrm{~mm}$. without harming the performance of the system). 
The material and fabrication cost for the other elements of the bearing system, for example the iron pole assemblies and the planar circuit array, should be quite small in large scale production, but it is beyond the scope of this report to attempt such a detailed cost analysis.

\section{IX) Losses of the Bearing System: General Comments}

Although it is not possible, in the absence of data from a fully engineered system, to specify the exact value of the losses associated with the bearing system described above, it is possible to make some estimates. These estimates, detailed below, indicate that with good design and quality control the bearing-related losses should be very low, of order a Watt or two. When we speak here of "losses" we are referring only to those losses that occur when the vehicle is parked and the bearing system is therefore not subject to any accelerations other than gravity. When in motion, particularly when the backup bearings come into play, the bearing losses could be much larger without influencing the overall performance, since under these conditions the energy flows into and out of the EMB are orders of magnitude larger than bearing-related losses.

There should be only two appreciable sources of losses within the system while it is parked. These are:

(1) Eddy-current and hysteresis losses in the pole faces of the attracting and repelling magnets associated with unbalance-related eccentricity of the rotation. (We believe that the use of permanent magnets embedded in iron pole structures will effectively eliminate any appreciable intrinsic azimuthal variation of the magnetic fields at the surface of these poles).

(2) Eddy-current losses in the conductors of the planar circuits of the axial stabilizers.

Evaluation of the first of these losses is difficult in the absence of a detailed analysis of a final design. However, a rough estimate will be given in terms of a simple analytical model. This estimate is favorable in that it predicts a low level of losses for reasonable values of the parameters.

Estimation of the second source of losses is easier, as the theory of eddy-current losses in litzendraht wires in a time-varying magnetic field is well known. These are the only appreciable losses within the 
axial stabilizers that should appear under standing conditions, that is, when they are not called on to deliver a net force.

\section{X) Losses of the Bearing System: Eccentricity-Related Sources}

We will base our estimate of the eccentricity-related losses in the bearing system on a very simple model. This model recognizes that only the time-varying component of the incident magnetic field at the surface of the magnet poles of either the attracting elements or the repelling elements contributes to eddy-current losses within those poles. The constant part of the field coming from the opposing pole will set up a fixed pattern of Amperian currents in the pole we are considering that accommodates to that flux. However, when the incident flux changes rapidly with time, the effect of eddy currents on the surface of the pole will be to provide an augmented current pattern that excludes the time-varying component of this flux from the interior of the pole.

To estimate the magnitude of the time-varying component of the flux we will approximate the magnitude of the once-per-revolution periodic field by simply considering the overlapping area between two annular rings with non-concentric axes. This assumption is a reasonable one when the gap between the two poles is very small. In such a case the magnetic flux through the pole will tend to vary linearly with the overlapping area. Thus the difference between the area of the poles and the overlapping area will be a measure of the magnitude of the periodic flux change at the surface of the pole, going to zero in the limit of zero eccentricity, and representing a linear function of the eccentricity. Each period of the rotation will then result in the dissipation by eddy currents of some fraction of the magnetic energy carried by the periodic component of the magnetic field.

For an eccentric displacement, $\varepsilon(\mathrm{m}$.$) , the ratio of the displaced$ area to the total pole area, with outer radius $\mathrm{r}_{0}(\mathrm{~m}$.$) and inner radius$ $r_{i}(m$.$) , is given by the relation:$

$$
\frac{\Delta \mathrm{A}}{\mathrm{A}}=\frac{2}{\pi}\left[\frac{\varepsilon}{\mathrm{r}_{0}-\mathrm{r}_{\mathrm{i}}}\right]
$$

Thus we find for the area $\Delta A$ the expression: 


$$
\Delta A=2 \varepsilon\left(r_{0}-r_{i}\right) \quad m^{2}
$$

Considering now the eddy-current losses in the poles, we note that the effect of eddy currents, which will be concentrated within a skin depth of the side and top surfaces of the poles, is to suppress flux variations within the pole. Thus, as mentioned above, the eddy currents will be of just such a magnitude as to produce an oppositely directed flux equal to the incident time-varying flux. The skin-depth within which these currents are concentrated is given by the expression:

$$
\delta=\sqrt{\frac{2 \rho}{\omega \mu}} \mathrm{m} .
$$

where $\rho(o h m-m$.$) is the resistivity of the pole material \omega$ is the frequency in radians/sec., and $\mu$ is the permeability (in MKS units).

The time-varying flux that is excluded by the surface eddy currents is approximately equal to the surface field, $\mathrm{B}_{s}$ times the nonoverlapping area of the poles, so that, using Equation 9, we can write for this flux:

$$
\Phi=2 \varepsilon\left(r_{0}-r_{i}\right) B_{s} \quad \text { Tesla-m } m^{2}
$$

It would be difficult to calculate the exact pattern of eddy currents needed to exclude this changing flux from the pole. However, for estimating purposes we can make a simplifying assumption, one that should not give grossly inaccurate answers. We therefor will assume that the eddy currents are confined to parallel strips, lying on the side surfaces of the pole, strips whose width is the order of the width of the pole face itself. We further assume that the currents in these strips produces a flux between them that is equal and opposite to the flux that is to be excluded. In this way we can arrive at an estimate of the magnitude of the actual currents, and thus of the resistive losses that they will introduce.

Integrating the flux between these two current strips at their plane of symmetry we are led to the following approximate expression: 


$$
\Phi=\frac{\mu_{0} I}{4 \pi}[\ln (5)+\pi]\left[\mathrm{r}_{0}+\mathrm{r}_{\mathrm{i}}\right]
$$

Equating the two fluxes we may solve for the current that flows in the skin-depth-deep strips, finding:

$$
I=\frac{8 \pi}{\mu_{0}[\ln (5)+\pi]}\left[\frac{r_{0}-r_{i}}{r_{0}+r_{i}}\right] \varepsilon B_{s} \quad \text { amperes }
$$

This current is flowing in the two conducting strips whose width is a skin depth and whose height is equal (by assumption) to the pole width, $\left(r_{0}-r_{i}\right)$. The total resistance of the ribbons is therefore equal to

$$
R=\left[2 \pi\left(r_{0}+r_{i}\right) \rho\right] /\left[\delta\left(r_{0}-r_{i}\right)\right]
$$

The power dissipated is equal to the rms value of $\mathrm{RI}^{2}$, so that we find for the estimated eddy current loss the expression:

$$
\mathrm{P}_{\mathrm{ec}}=\left[\frac{64 \pi^{3}}{\ln (5)+\pi}\right]\left[1 / \mu_{0}^{2}\right]\left[\frac{\mathrm{r}_{\mathrm{o}}-\mathrm{r}_{\mathrm{i}}}{\mathrm{r}_{\mathrm{o}}+\mathrm{r}_{\mathrm{i}}}\right]\left[\varepsilon^{2} \rho \mathrm{B}_{\mathrm{s}}^{2} / \delta\right) \quad \text { Watts }
$$

Putting in the numerical value of the constants yields the equation:

$$
P_{e c}=2.6 \times 10^{14}\left[\frac{r_{0}-r_{i}}{r_{0}+r_{i}}\right]\left[\varepsilon^{2} \rho B_{s}^{2} / \delta\right) \quad \text { Watts }
$$

Inserting the resistivity of iron, $1.0 \times 10^{-7} \mathrm{ohm}-\mathrm{m}$, and assuming a permeability of $1000 \mu_{0}$ for the iron pole material, and a frequency of $1000 \mathrm{~Hz}$, we find for the skin depth the value:

$$
\delta=1.6 \times 10^{-4} \mathrm{~m} .
$$

so that our expression for the eddy-current losses (with iron as the conducting material) becomes:

$$
\mathrm{P}_{\mathrm{ec}} \text { (iron) }=1.6 \times 10^{11}\left[\frac{\mathrm{r}_{\mathrm{o}}-\mathrm{r}_{\mathrm{i}}}{\mathrm{r}_{\mathrm{o}}+\mathrm{r}_{\mathrm{i}}}\right]\left[\varepsilon^{2} \mathrm{~B}_{\mathrm{s}}^{2}\right] \quad \text { Watts }
$$


Taking the repelling poles as an example, with $\mathrm{r}_{\mathrm{o}}=.06 \mathrm{~m}$. and $\mathrm{r}_{\mathrm{i}}=$ $.054 \mathrm{~m} ., \mathrm{B}_{\mathrm{s}}=0.5 \mathrm{Tesla}$, and $\varepsilon=3 \times 10^{-5} \mathrm{~m}\left(.001^{\prime \prime}\right.$, typical of what has been found in practice when care has been taken in the balancing), we find for the power loss (per pole face) the value

$$
\mathrm{P}_{\mathrm{ec}} \text { (iron) }=1.9 \mathrm{Watts} / \text { pole }
$$

This level of losses would be marginally acceptable, but the approximate nature of the calculation is such that there is possible cause for concern. Note also that because the losses vary as the square of the eccentricity, the effect of poor balance (larger values of $\varepsilon$ ) in increasing the loss is magnified.

If we now re-examine the expression for the eddy current losses it can be seen that there is available a simple way to reduce the losses substantially. This way would be to copper plate the iron pole faces to a thickness that is comparable to the skin depth in copper. If we now recalculate the constants in Equation 16, inserting the resistivity of copper, we find for $\delta$ the value $1.5 \times 10^{-3} \mathrm{~m}$., and for the losses the expression:

$$
\mathrm{P}_{\mathrm{ec}}(\text { copper })=3.0 \times 10^{9}\left[\frac{\mathrm{r}_{\mathrm{o}}-\mathrm{r}_{\mathrm{i}}}{\mathrm{r}_{\mathrm{o}}+\mathrm{r}_{\mathrm{i}}}\right]\left[\varepsilon^{2} \mathrm{~B}_{\mathrm{s}}^{2}\right] \quad \text { Watts : }
$$

The eddy current loss calculated for the previous case now is greatly reduced, becoming

$$
\mathrm{P}_{\mathrm{ec}}(\text { Copper })=35 \text { milliWatts } / \text { pole }
$$

This low a level of losses, when summed over all the poles, would still represent an almost negligible energy drain on the system during standing.

Another method of reducing eddy-current losses in the poles, mentioned in Section $V$, is to scale up the bearing size, and then to operate at a larger dimensionless gap. This procedure can preserve the same magnitude of the force derivative, while at the same time reducing the relative amount of eccentricity-induced flux change by reducing the magnetic gradients at the surface of the poles. 
In the above examples, where the flux is excluded from the iron pole faces by the shielding eddy currents, and where there will be present a very low value of the alternating flux component as compared to the main field, losses from magnetic hysteresis should be completely negligible. In case the iron or the copper-plated iron poles were to be replaced by powdered iron pole material there would be a very small loss from hysteresis effects, but in this case there would be totally negligible losses from eddy currents, owing to the elimination of macroscopic conducting surfaces.

The conclusion to be drawn from the above discussion about the losses in the permanent-magnet excited elements of the bearing system is that there exist design concepts that should lead to satisfactorily low losses from that part of the system. We turn now to the second source of losses under static conditions, the eddy currents in the litzendraht wires of the planar circuits in the axial stabilizers.

\section{XI) Losses of the Bearing System: The Axial Stabilizers}

Under equilibrium conditions, when the planar circuit of the axial stabilizer is centered midway between the two Halbach arrays, there should be near-zero current in its conductors and thus negligible ohmic losses in these conductors. However, the conductors will be immersed in a strong time-varying magnetic field so that an eddy current pattern will develop within these conductors that will entail losses. The classic method of reducing these losses is the use of litzendraht wire, that is, a conductor made up of a bundle of insulated fine wires, specially braided so that each wire in that conductor experiences the same average flux (thereby minimizing "closed-loop" currents from end to end of the conductors). As will be seen, the use of sufficiently finely divided litz wire in the Halbach array can reduce this source of losses to a very small value, one that is completely compatible with the rundown-time requirements of the bearing system.

From theory we have a simple expression for the eddy-current losses, in units of Watts/meter, for a thin wire of radius, a (m.) and resistivity $\rho$ (ohm-meters), immersed in a magnetic field B (Tesla), varying at an angular frequency of $\omega$ (radians/sec/). The expression is:

$$
\mathrm{P} / \mathrm{L}=\frac{\pi}{8}\left[\frac{\mathrm{B}^{2} \omega^{2} \mathrm{a}^{4}}{\rho}\right] \quad \text { Watts/meter }
$$


We will now apply this relationship to the planar circuit array described in Section VIII. In that circuit array there are 48 radially directed conductors, each about .025 meters long, for a total length of conductor immersed in the rotating Halbach field of $1.2 \mathrm{~m}$. The magnitude of this field at a distance $h(m$.) from the surface of the magnets (i.e., half the separation between the two Halbach arrays) is given by the relation:

$$
\mathrm{B}=2.0 \mathrm{~B}_{0} \exp (-2 \mathrm{kh}) \quad \text { Tesla }
$$

Here $\mathrm{k}=80 \mathrm{~m}^{-1}, \mathrm{~h}=.006 \mathrm{~m}$., and $\mathrm{B}_{0}=1.0$ Tesla, so that $\mathrm{B}=0.77$ Tesla.

Litz wire can be obtained commercially in a variety of sizes. For our example design we will choose a conductor that is equivalent to 16 AWG wire in conductivity, being made up of 1000 strands of 46 AWG copper wire. The radius of each 46 AWG strand is $2.0 \times 10^{-5} \mathrm{~m}$. The total length of these strands exposed to the field is $1000 \times 1.2=1200$ m. From Equation 19 we then find for the eddy-current loss the result

$$
P=2.6 \times 10^{-9} \omega^{2} \quad \text { Watts }
$$

At a rotation speed of $1000 \mathrm{~Hz}(60,000 \mathrm{RPM})$, we find for the eddy-current loss the value 0.10 Watts. Because this level of losses is much lower than our overall target of $P_{\text {loss }}<5$ Watts it would be possible to use a larger gauge litz wire (if this was desirable). For example, in case we wished to achieve a lower transition speed or if we wished to reduce resistive heat losses when the stabilizer is required to produce substantial forces in the presence of transient acceleration loads.

\section{XII) Other Technical Issues}

In the foregoing we have described the main details of the design of the magnetic elements of a passive bearing system. To be fully operative, that is to provide for operation from zero speed up to the "transition speed" of the axial stabilizers, and for axial and radial restraint of the rotor in the presence of accelerations in excess of $1.0 \mathrm{~g}$ (the design limit for the magnetic bearings assumed in the example given here), as noted, mechanical backup bearings must be used. Also, as in all high-speed rotating systems, damping, either in the form of eddy-current-based dampers, or viscous mounting of non-rotating bearing components, needs to be provided. These techniques are well- 
known in the art and therefor will not be discussed here, important though they are.

\section{XIII) Summary and Conclusion}

In this report an approach is described to the problem of designing a passive magnetic bearing system suitable for use in an electromechanical battery in a hybrid-electric vehicle. The main requirements that a magnetic bearing system for such service must address are those associated with acceleration-induced loads, those coming from the need for low losses while the vehicle is parked, and those associated with minimizing cost. We believe that the design approaches that have been suggested can meet all of the above requirements.

Better to define the approach, a "straw man" design has been presented. This example could be used as a starting point for the design of a practical system. The design utilized primary data on some large magnetic bearing elements that were derived from magnetics computer-code calculations. These data were then transformed to the smaller size appropriate to the straw man case by using scaling laws given in this report.

While this report has emphasized the application of the ideas presented to hybrid-electric vehicles, the approach described here should have applications within the whole spectrum of uses for electromechanical batteries. It might also have applications in other areas of use of magnetic bearings. In particular, the ability to passively levitate with minimal losses at high rotation speeds suggests that these bearing systems could compete favorably with magnetic bearing systems utilizing superconductors. In stationary applications; for example, larger EMBs for load-leveling applications, stiffness requirements could be greatly relaxed. In such situations, as the analyses presented here can demonstrate, the losses of the entire bearing system might be reduced to substantially less than 1 Watt. A 1 Watt bearing loss level for a $10 \mathrm{kWh}$ EMB rotor would correspond to a run-down time of more than 1 year! Unlike either superconducting bearings or active magnetic bearing systems, our passive magnetic bearings have no external power "overhead" that must be supplied at all times, either in the form of cryogenic refrigeration for the superconducting bearings or power dissipated in electronic amplifiers in active magnetic bearing systems. 


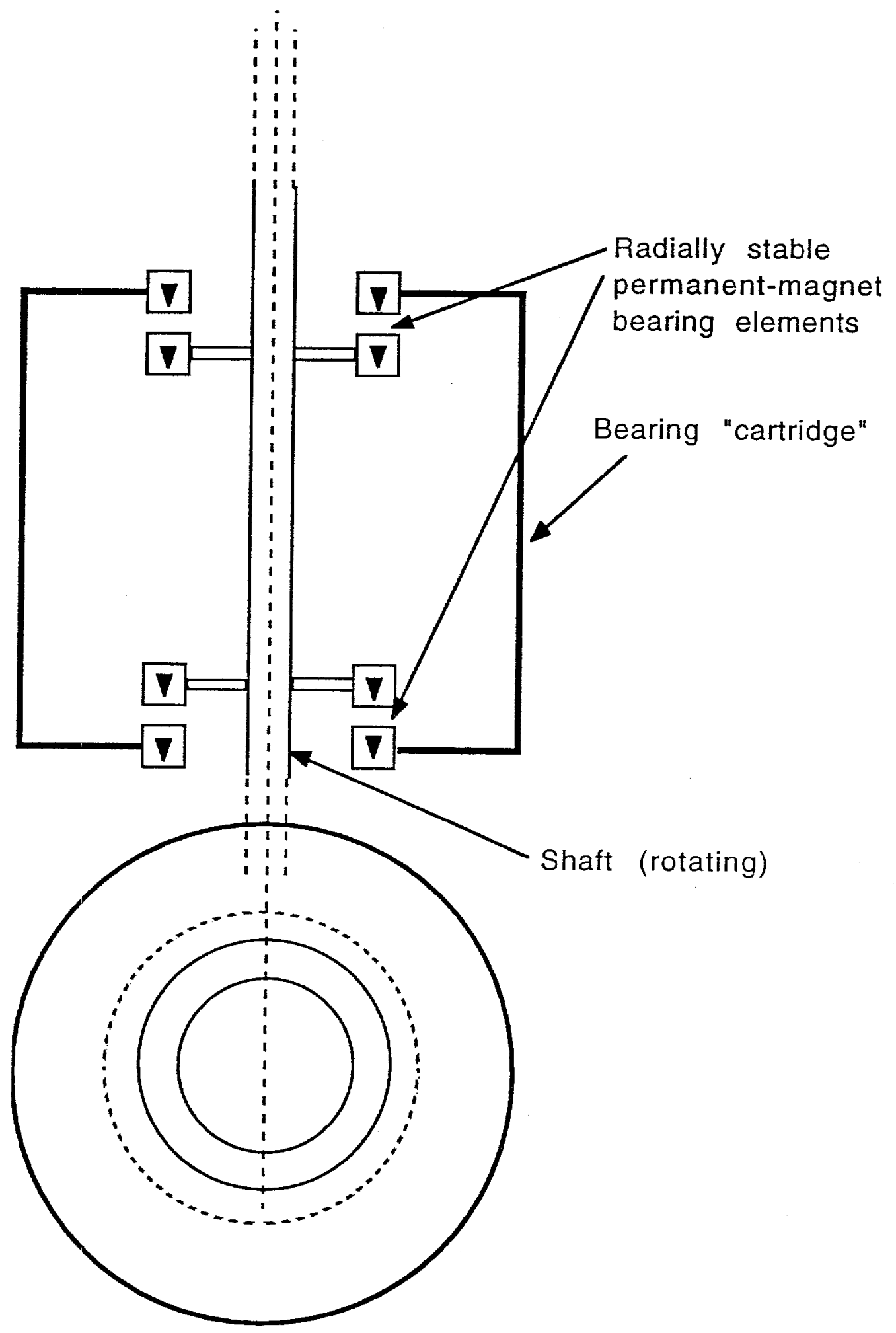

Figure 1a 


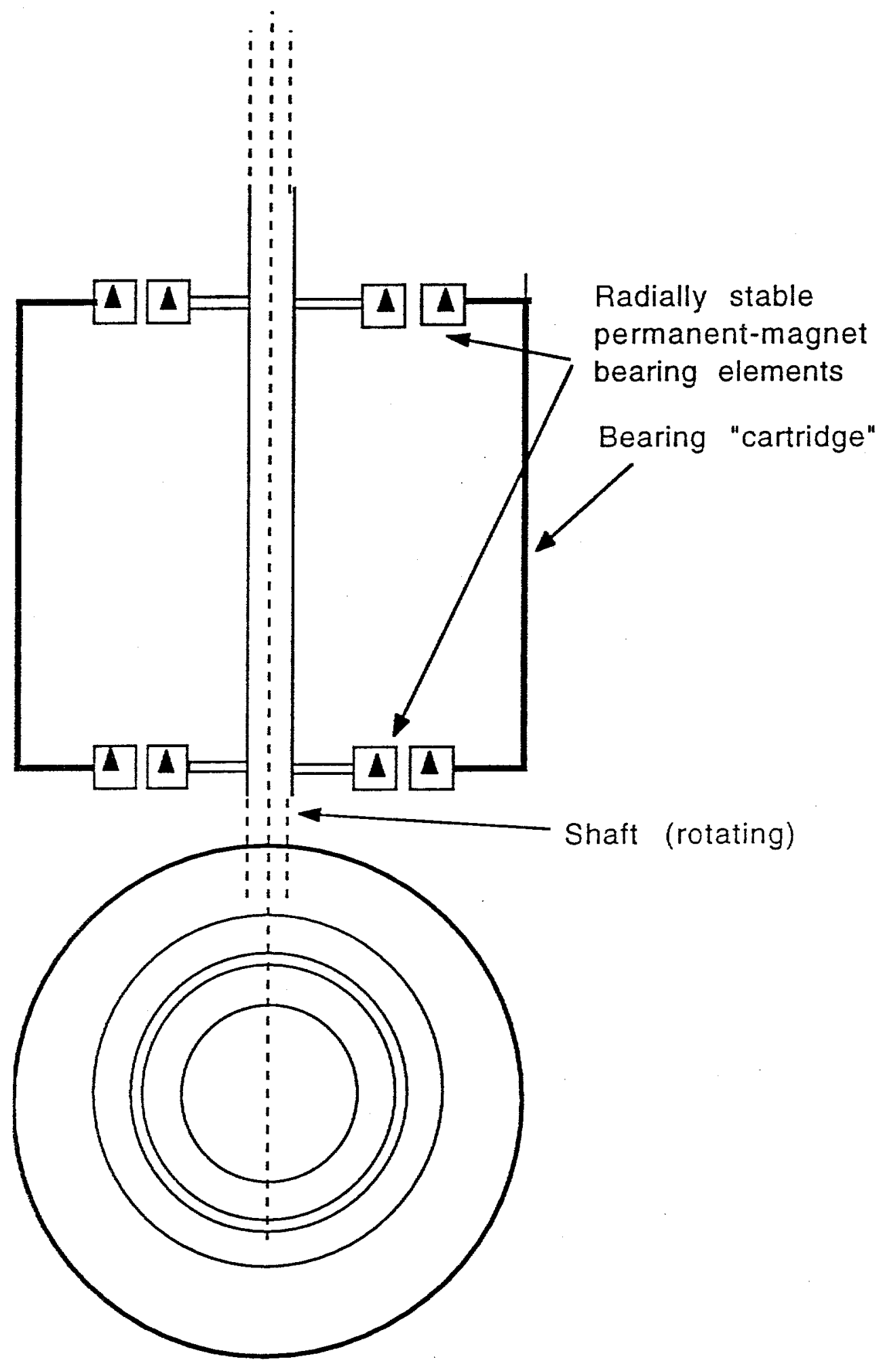

Figure $1 b$ 

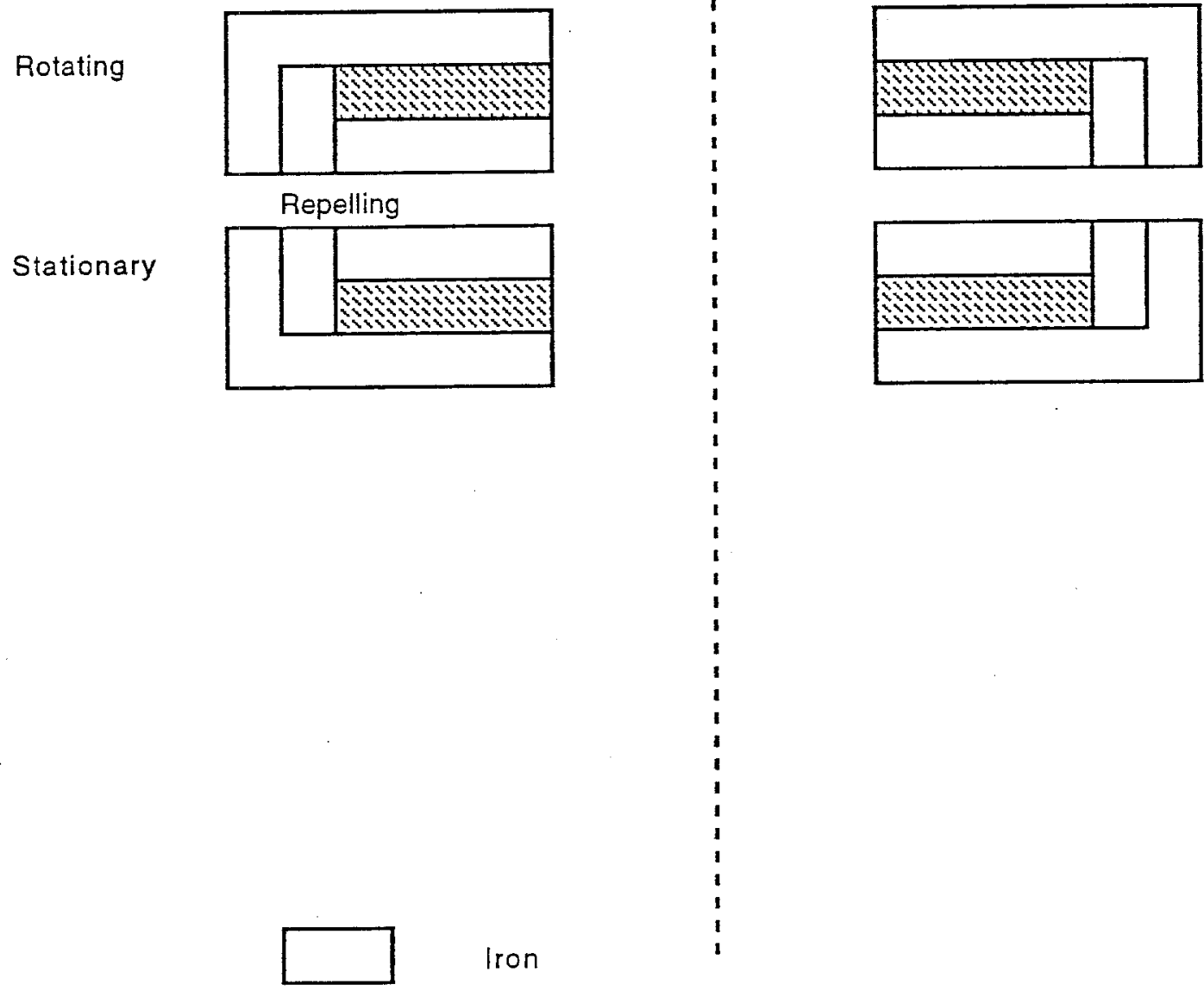

NdFeB permanent magnet material

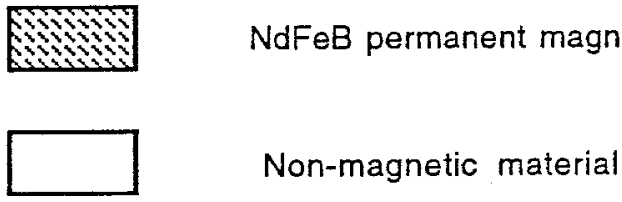

Figure 2 


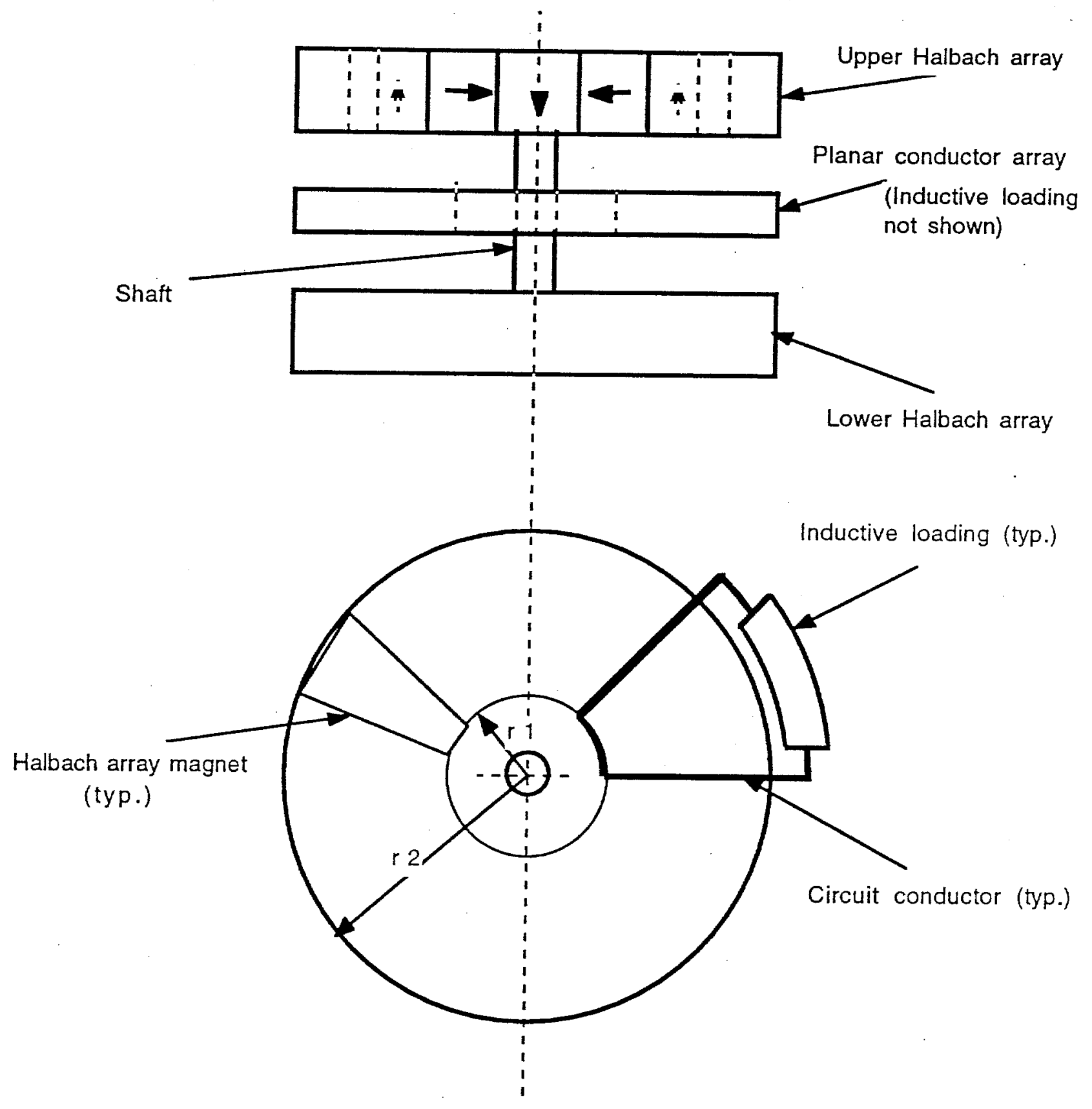

Figure 3 


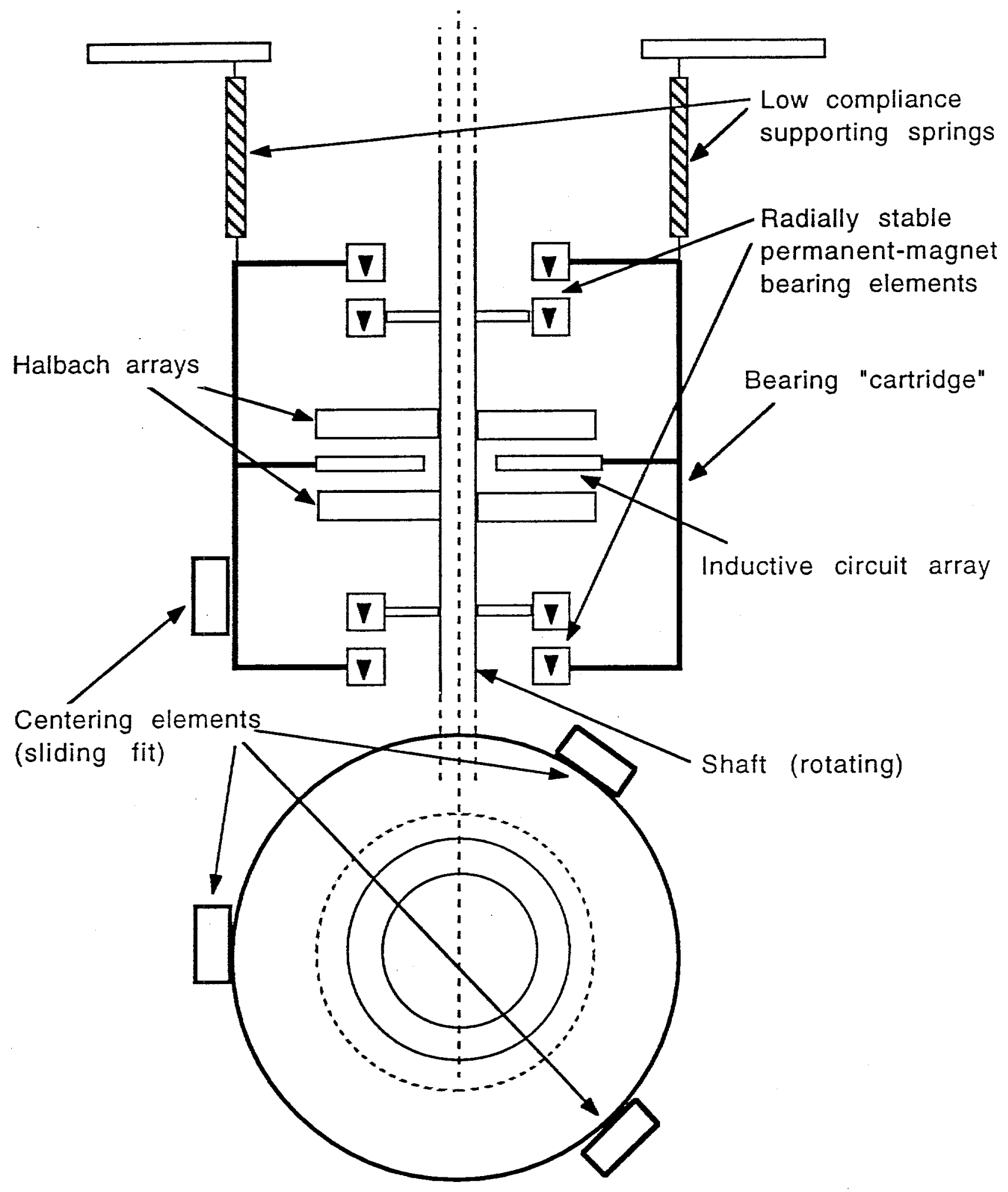

Figure 4 


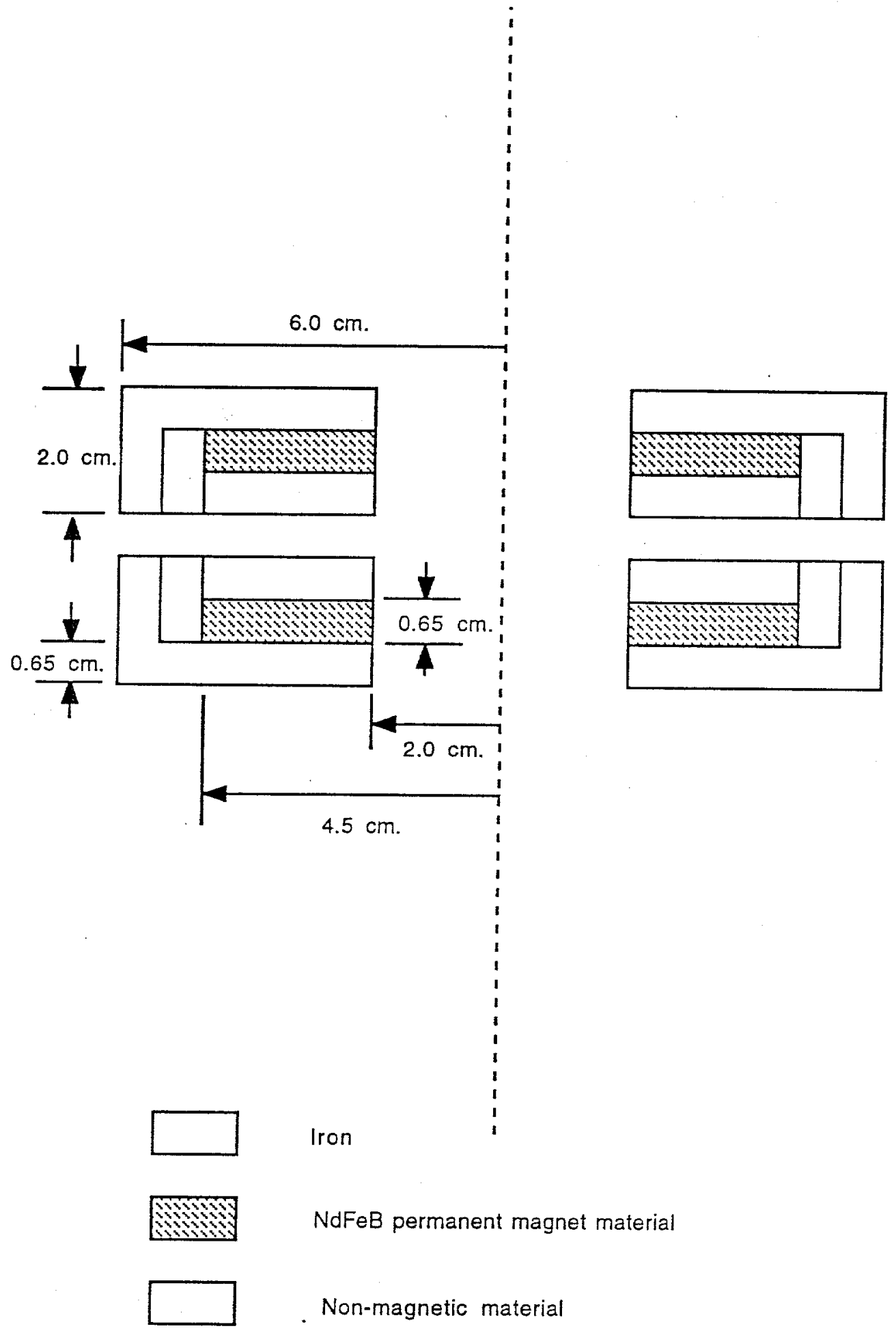

Figure 5 
Attracting Force vs Displacement

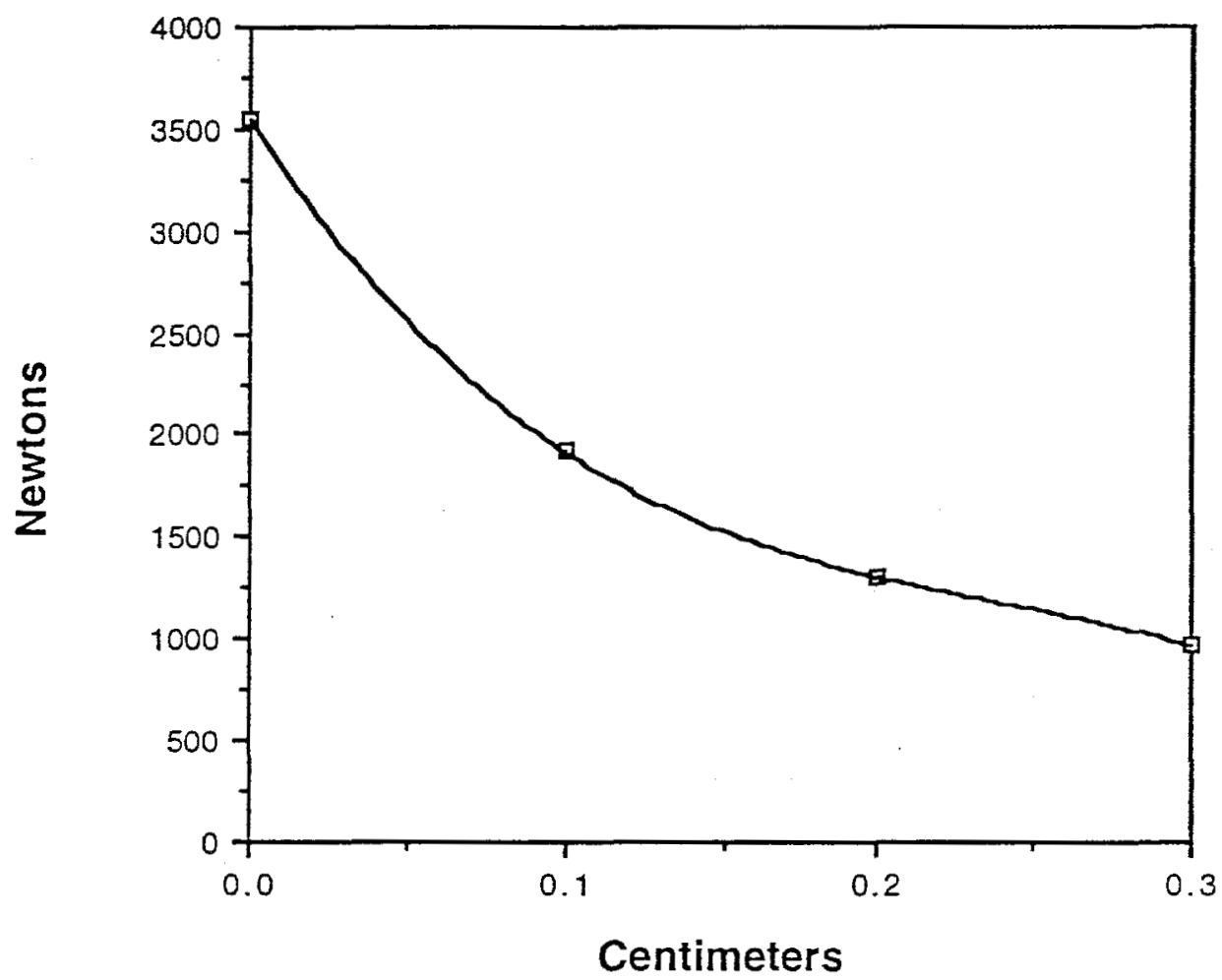

Figure 6 
Repelling Force vs Displacement

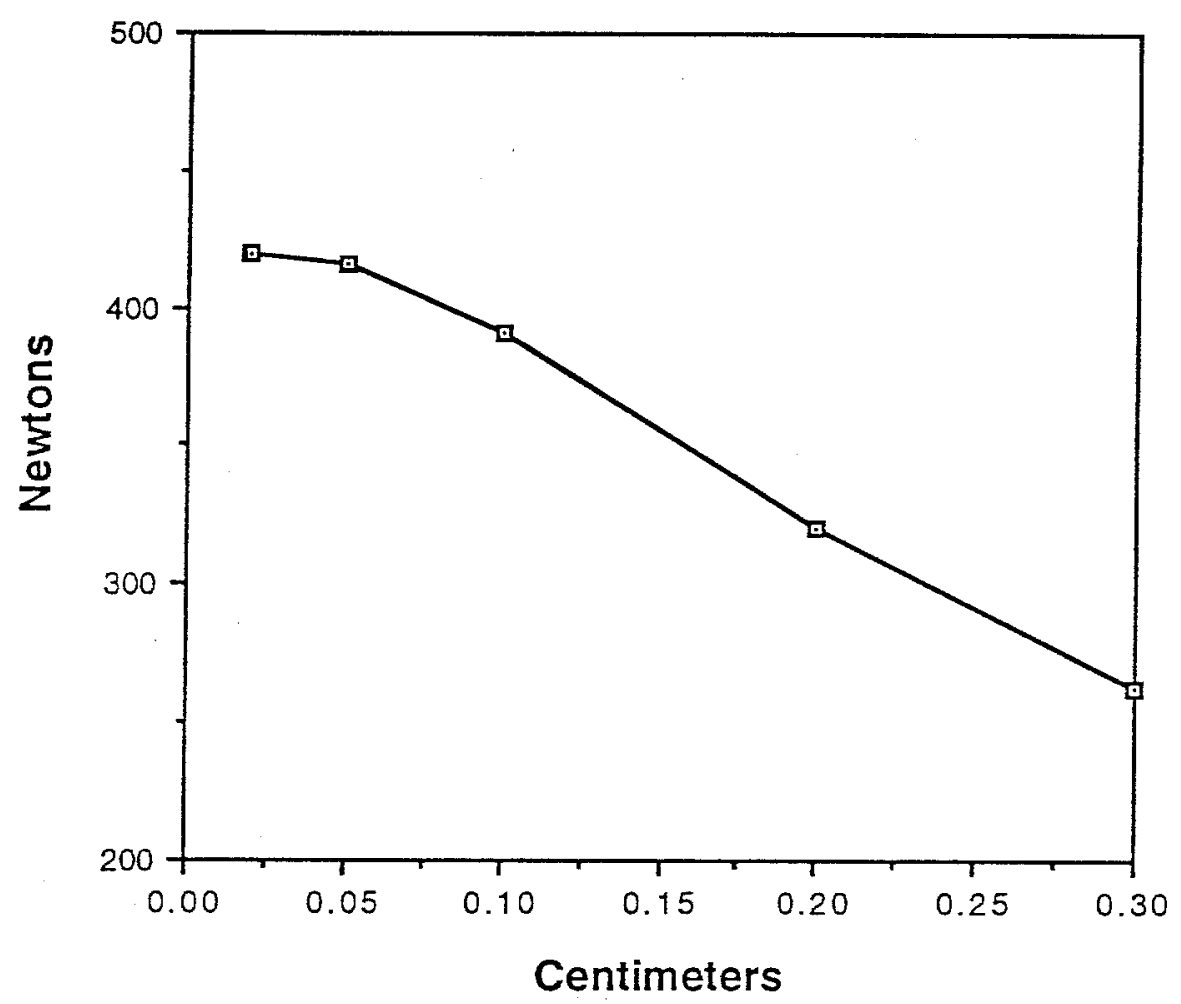

Figure 7 


\section{Figure Captions}

Figure 1a: Schematic representation of the centering elements of a magnetic bearing cartridge: Stacked annular elements

Figure 1b: Schematic representation of the centering elements of a magnetic bearing cartridge: Nested annular elements

Figure 2: Annular poles with permanent-magnet excitation

Figure 3: Schematic representation axial stabilizer consisting of two (upper and lower) planar Halbach arrays with inductively loaded planar circuits between the arrays.

Figure 4: Schematic drawing of a complete bearing cartridge with its annular centering magnets, its axial stabilizer, and its vertically compliant supports

Figure 5 Annular poles as in Figure 2, but with dimensions shown

Figure 6 Plot of the calculated axial force vs displacement (zero displacement corresponds to contact) of a pole assembly as shown in Figure 5: Attracting case

Figure 7 Plot of the calculated axial force vs displacement (zero displacement corresponds to contact) of a pole assembly as shown in Figure 5: Repelling case 
Distribution List:

Ken Fowler L-640

Dimitri Ryutov L-640

Ray Smith L-640

LouAnn Tung L-418

John Wooldridge L-703

Don Bender

Trinity Flywheel Batteries, Inc.

10 Lombard Street, Suite 410

San Francisco, CA 94111

Mike Bowler

Trinity Flywheel Batteries, Inc.

P.O. Box 1056

Edmonds, WA 98020

Vault Reference (3 copies)

OSTI ( 2 copies)

Program File (3 copies) 